\title{
Optimization of Oil Adsorption Capacity by Aerogel Powder Synthesized Using Emulsion Droplets as Micro-reactors in Ambient Conditions from Sodium Silicate as Precursor
}

\author{
Young-Sang Cho ${ }^{1, *}$, Sohyeon Sung ${ }^{1}$, Seung Hee Woo ${ }^{1}$, and Young Seok Kim ${ }^{2}$ \\ ${ }^{I}$ Department of Chemical Engineering and Biotechnology, Korea Polytechnic University, Gyeonggi 15073, Republic of Korea \\ ${ }^{2}$ Display Components \& Materials Research Center, Korea Electronics Technology Institute, Gyeonggi 13509, Republic of Korea
}

\begin{abstract}
In this study, silica aerogel particles were synthesized from emulsion droplets as micro-reactors at room temperature under ambient pressure. An economical precursor, sodium silicate, was used as the starting material for silica, and an emulsification technique was applied to form droplets in continuous phase. By controlling the composition of the dispersed phase using ammonium hydroxide, the effect of $\mathrm{pH}$ on the morphologies of the final aerogel particles was studied by SEM observation. As a demonstrative application, hydrophobic silica aerogel particles were produced by modification using a silane coupling agent, for oil adsorption. The amount of oil adsorbed by the aerogel particles was optimized by adjusting the concentration of precursors in the emulsion droplets, the composition of the dispersed phase, and the concentration of the coupling agent during surface treatment of the particles. The resulting aerogel particles were characterized using BET, TGA, and the contact angle of water droplets after modification using silane coupling agents with different carbon numbers. The optimized value of adsorbed silicone oil (100 CS) was measured to be about 250 $\%$ relative to the weight of the aerogel particles. For comparison, other types of porous silica particles were also prepared from emulsion-assisted self-assembly routes to quantify the amount of adsorbed oil.
\end{abstract}

(Received October 5, 2020; Accepted December 24, 2020)

Keywords: porous particles, aerogel powder, complex fluid, oil adsorption

\section{Introduction}

Porous materials have attracted wide attention because of their useful functionalities and microstructures for various applications in including catalytic supports, thermal insulators, sound absorbers, adsorbents, energy materials, and separation membranes [1-6]. For several decades, the synthesis routes of porous materials have typically involved chemical approaches, such as the hydrothermal method, sol-gel method, and gasphase synthesis like the spray pyrolysis method [7-10].

Among porous materials, aerogels are considered promising materials for construction or electronic engineering, as insulators, as well as supporting structures for catalytic materials [11-13]. Although many studies have been conducted on aerogel materials, most commercial aerogel

- 조영상: 부교수, 성소현: 석사과정, 우승희: 학사과정, 김영석: 연구원

*Corresponding Author: Young-Sang Cho

[Tel: 82-31-8041-0612, E-mail: yscho78@kpu.ac.kr]

Copyright (c) The Korean Institute of Metals and Materials synthesis has been carried out busing the supercritical drying approach under harsh conditions. It would be highly desirable to develop a novel aerogel synthesis method that could be performed at room temperature under ambient pressure [14]. Emulsion-assisted self-assembly combined with the sol-gel method may be a potential alternative approach to synthesize an aerogel material in particulate form. The tiny droplets produced at room temperature can be employed as micro-reactors for the chemical synthesis of porous materials [15].

Recently, oil contamination from ships and automobiles has caused serious environmental problems, since artificially synthesized oils as well as crude oil are hazardous materials to human health and wildlife. Several studies on ways to remove oils have been carried out utilizing non-woven fabrics for adsorption, the extraction of target liquids, and adsorption by proper particulate materials [16-18]. Among them, the adsorption of oil by particles is advantageous in that it permits the facile separation of the oil-particle mixture, 
since solid-like behavior of the mixture can be expected [19]. However, further research is still necessary to maximize the amount of oil adsorbed by optimizing various synthesis conditions using adequate particulate materials such as porous particles or aerogels.

In the present article, silica aerogel particles were synthesized from emulsion droplets as micro-reactors under ambient pressure and room temperature. During synthesis, the energy cost of the high temperature and pressure was not comparable to that of supercritical drying. After surface modification using a silane coupling agent, a hydrophobic aerogel powder was fabricated for the adsorption of silicone oil. Various synthesis parameters such as the amount of ammonia, the concentration of reactants, and the amount of silane coupling agent were investigated to optimize the amount of adsorbed silicone oil. During surface modification, the effect of hydrocarbon chain length on the silane coupling agent was also studied by measuring the amount of silicone oil adsorbed. For comparison, silica microparticles with wrinkled surfaces as well as porous silica microparticles were synthesized using emulsion-assisted self-assembly, for application to oil adsorption.

\section{Materials and Methods}

\subsection{Materials}

The silica precursor, sodium silicate, was bought from Samchun Chemicals, and purified using anion exchange resin (Amberlite IR 120, Sigma-Aldrich). Ammonium hydroxide (25-30\%, Duksan Chemicals) was mixed with the resulting silicic acid solution to adjust the $\mathrm{pH}$ of the mixture. For emulsification, tetradecane was procured from Beyond Industry limited and used in continuous phase. Abil EM90 was used as the emulsifier and bought from Cosnet. TEOS (tetraethylorthosilicate, 99.9\%) was adopted as another silica precursor and was procured from Sigma-Aldrich.

To modify the surface of the aerogel particles, silane coupling agents such as trichlorophenylsilane, trichlorooctadecyl;silane, trichlorodododecylsilane, trichlorooctylsilane, trichlorohexylsilane, trichlorobutylsilane, and trichloromethylsilane were purchased from Sigma-Aldrich.

For emulsion polymerization, styrene used as monomer was bought from Daejung Chemicals. Initiator, potassium persulfate (KPS) was purchased from Kanto Chemicals. Comonomer, 4-styrenesulfonic acid sodium salt hydrate (NaSS) was procured from Aldrich Chemicals.

\subsection{Synthesis of aerogel Particles from Emulsion Droplets as Micro-reactors}

Sodium silicate diluted with water at a 2:1 mixing ratio was purified by removing sodium ions using an anion type ion exchange resin. Then, the resulting silicic acid solution was mixed with a fixed amount of ammonium hydroxide solution to prepare the dispersed phase. Emulsification was performed to produce emulsions droplets after adding the dispersed phase to the continuous phase (tetradecane) at a mixing ratio of 1:3 using homogenizer (HG-15a-set-a, Daihan) at about $900 \mathrm{rpm}$. To prevent the droplets from coalescence, a small amount of emulsifier, Abil EM90 was dissolved in the tetradecane before emulsification. Gelation of the silicic acid inside the droplets was performed under mild stirring at room temperature for one day. After stopping the stirring and gravitational sedimentation of the resulting particles, the tetradecane was removed from the particles, followed by washing with hexane three times. The washing steps were repeated with ethanol and water to remove ammonium hydroxide and residual reactants from the aerogel particles.

To avoid shrinkage of the particles' porous structure, an additional solvent exchange was carried out with ethanol and toluene. Then, the surface hydroxyl groups of the particles were replaced with hydrocarbon molecules by mixing the particles with a solution of a silane coupling agent, such as trichlorododecylsilane dissolved in toluene. During surface modification, sedimented particles were collected after the careful removal of supernatant liquid. The resulting hydrophobic aerogel particles were dried at room temperature to measure the amount of oil adsorbed by the aerogel particles.

\subsection{Synthesis of Silica Particles with Wrinkled Surfaces}

Aqueous silicic acid solution was mixed with additional water at the proper mixing ratio, followed by emulsification using a homogenizer. Then, the resulting complex fluid was heated at $95^{\circ} \mathrm{C}$ to induce a gelation reaction on the droplets' interface. Then, tetradecane was removed from the sedimented 
particles, followed by washing with hexane and drying at room temperature. The hydrophilic surface of the resulting silica particles was modified using the silane coupling agent. Detailed conditions of the particles synthesis and surface modification can be found elsewhere [20,21].

\subsection{Synthesis of Porous Silica Particles with Wrinkled Surfaces}

An aqueous suspension of polystyrene (PS) nanospheres synthesized by emulsion polymerization was mixed with the aqueous silicic acid solution. As with the silica particles with wrinkled surfaces, emulsification, heating, and washing steps were carried out to obtain composite particles of silica and PS nanospheres. Then, calcination of the composite particles was performed at $500{ }^{\circ} \mathrm{C}$ for 5 hours to produce porous silica particles with wrinkled surfaces, followed by surface modification using the silane coupling agent for hydrophobic particles.

\subsection{Oil adsorption by Porous Silica Particles}

Silicone oil (100 CS) was adsorbed by the porous silica particles, including the aerogel particles, particles with wrinkled surfaces, and porous particles with wrinkled surfaces. Fixed amounts of the silica particles were added to a beaker, followed by the stepwise addition of the oil. As the oil was added, the silica particles and the oil were mixed uniformly using a spoon, and the addition of oil was stopped when the liquid oil started to infiltrate from the mixture of oil and silica powder. When this termination point was reached, the added amount of oil was recorded to measure the amount of adsorbed oil. Until the termination point, the behavior of the mixture of oil and silica powder was like a solid rather than a liquid.

\subsection{Characterizations}

The morphologies of the porous silica particles were observed using a field emission scanning electron microscope (FE-SEM, Hitachi-S4700). The composition change after the surface modification of the porous silica particles was analyzed using an FT-IR spectrometer (Nicolet, Thermo Fisher Scientific co. Ltd). The surface area and pore sizes of the silica aerogel particles were measured using BET equipment (ASAP-2010). Thermogravimetric analysis (TGA) of the silica aerogel particles was carried out using a Thermal Analysis System (TG-8120). The contact angle of the deposited film with the silica aerogel particles was measured using a contact angle measurement system (Phoenix-Mini, Surface \& Electro-Optics Co. Ltd.). The ice adhesion strength of composite films composed of PDMS, silica aerogel particles, and silicone oil was measured using a lab-made instrument. The instrument was prepared in an ice box to apply a shear force to the ice layer adhered on the film. After freezing at -20 to $-25{ }^{\circ} \mathrm{C}$ for 30 to 40 minutes, an icing area of $0.1 \mathrm{~cm}^{2}$ was pulled at $0.5 \mathrm{~mm} / \mathrm{s}$ until the layer was detached, to measure ice adhesion strength.

\section{Results and Discussions}

In this study, porous silica particles were synthesized from emulsion droplets as micro-reactors using sodium silicate as the precursor. Silica aerogel microparticles with spherical morphologies could be synthesized by adjusting the mixing ratio of silicic acid and ammonium hydroxide for the gelation of the silica precursor inside the emulsion droplets. After removing the remaining reactants inside the particles by washing, solvent exchanges using ethanol and toluene were applied to maintain the porous structure of the aerogel particles, followed by surface modification with a silane coupling agent like trichloromethylsilane for hydrophobic aerogel particles, as depicted schematically in Fig. 1(a). To compare oil adsorption capacity, silica microparticles with wrinkled surfaces were also synthesized from emulsion droplets, without using ammonium hydroxide. In this case, emulsion droplets containing silicic acid was heated for preferential gelation on the droplets' interface, causing crumpling and folding of the microparticles during droplet shrinkage. The resulting wrinkled surface was also modified using the silane coupling agent used for the hydrophobic particles, as described in Fig. 1(b). For some samples, sacrificial templates such as polystyrene nanospheres were included during synthesis of the silica microparticles with wrinkled surfaces, to create macroporous structures inside the silica microparticles, as described in Fig. 1(c). After surface modification of these porous silica particles with wrinkled surfaces, the amount of adsorbed silicone oil was compared with the results for other types of silica microparticles. 

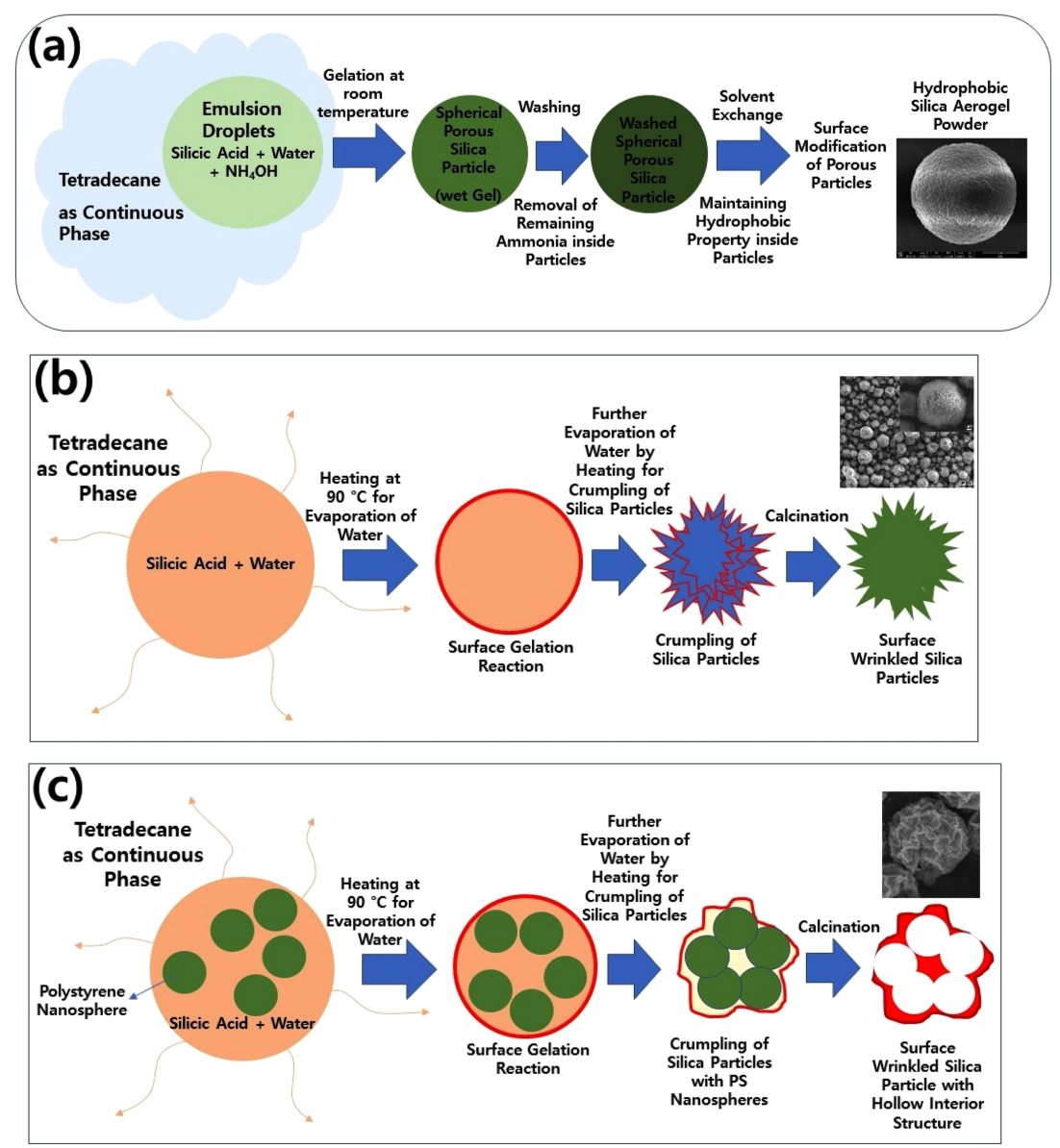

Fig. 1. Schematic figure for the synthesis of (a) silica aerogel microparticles, (b) silica microparticles with wrinkled surfaces, and (c) silica microparticles with wrinkled surfaces and hollow interior structure from emulsion droplets as micro-reactors.

In this study, silane coupling agents with hydrocarbon groups were adopted to change the surface property of silica microparticles. During surface modification, the hydroxyl groups on the silica particle surface can be changed by the hydrocarbon chains in the silane coupling agent by the alcoholysis reaction, as described schematically in Fig. 2(a). The change in functional groups during surface modification was confirmed by FT-IR analysis shown in Fig. 2(b). After treatment using a coupling agent like trichlorododecylsilane, characteristic peaks near $3,000 \mathrm{~cm}^{-1}$ appeared due to dodecyl groups on the silica surface, indicating that a hydrophobic property could be imposed on the silica aerogel particles. When heated from 300 to $600{ }^{\circ} \mathrm{C}$, the characteristic peaks due to the dodecyl groups disappeared, leaving silica aerogel particles with the FT-IR absorption spectrum of conventional silica, as shown in the graph in Fig. 2(b).

During the synthesis of the silica aerogel particles, the volume ratio of silicic acid solution and ammonium hydroxide was adjusted from $5: 1$ to $10: 1$, and the morphologies of the resulting particles are displayed in the SEM images of Fig. 3. When the amount of ammonium hydroxide was a relatively small value, such as the mixing ratio of 5:1, the morphologies of the silica aerogel particles were irregular shapes, as shown in Fig. 3(a), indicating that the gelation speed was not fast enough to maintain the spherical morphologies of the original emulsion droplets. Thus, small silica particles aggregated to form larger irregular particles. When the amount of ammonium hydroxide was increased to a mixing ratio of 7:1, disk-shaped and spherical silica aerogel particles were formed together, indicating that the rigidity of the aerogel particles may not be strong enough to maintain the spherical morphologies, deforming the spherical microparticles into oblate ellipsoids. In this case, the gelation of silica sol proceeded completely, since microparticles could be formed from emulsion droplets 

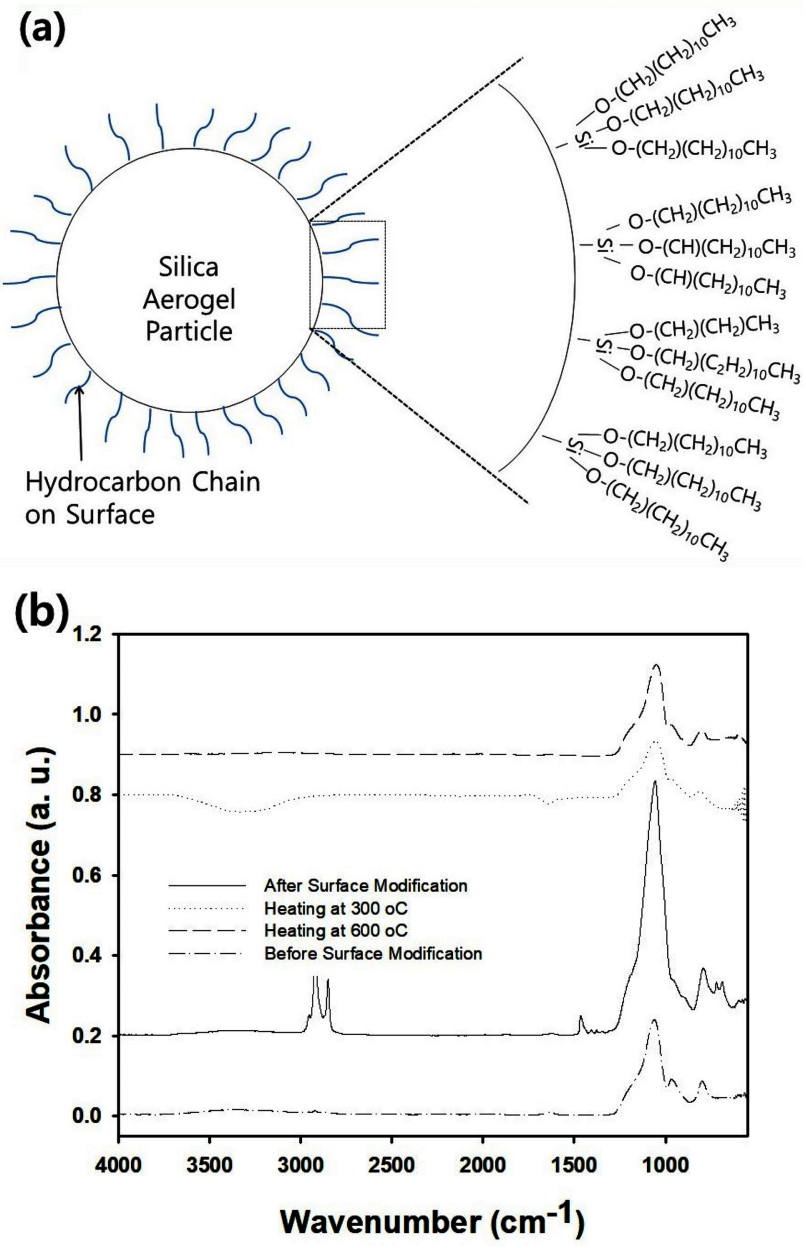

Fig. 2. (a) Schematic figure for the surface modification of silica microparticles using hydrophobic silane coupling agent. (b) FT-IR spectrum of silica aerogel microparticles before and after surface modification using trichlorododecylsilane. The spectra after surface modification were also included in the graph.

as micro-reactors, as displayed in SEM image of Fig. 3(b).

Further increase in ammonium hydroxide to a mixing ratio of 9:1 resulted in the formation of spherical aerogel particles, as shown in the SEM image of Fig. 3(c). Since the amount of ammonia was sufficient to promote fast gelation inside the emulsion droplets and produce strong rigidity, all of the aerogel particles exhibited a spherical morphology. The $\mathrm{pH}$ of the dispersed phase according to the mixing ratio of silicic acid and ammonium hydroxide is summarized in Table 1,
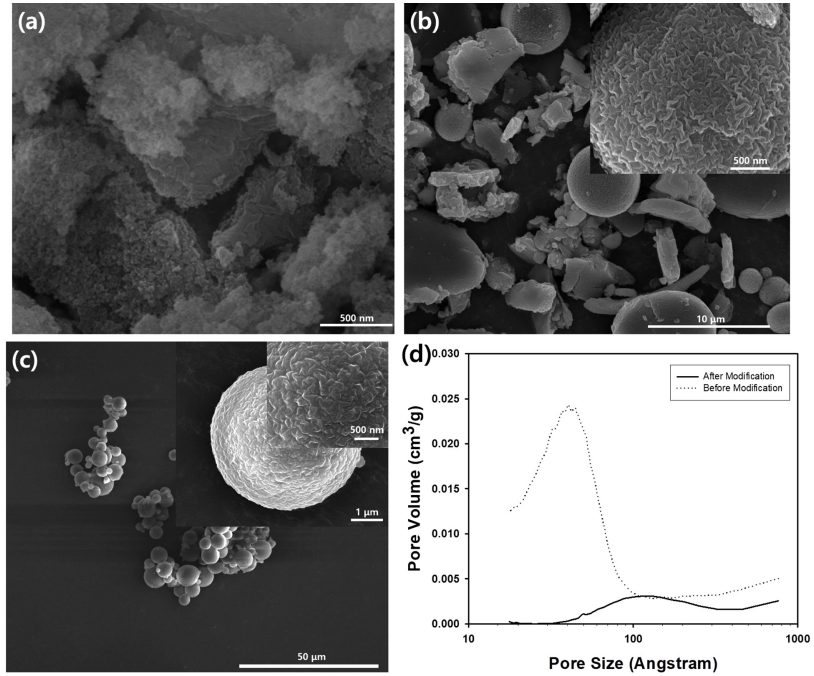

Fig. 3. SEM image of silica aerogel microparticles synthesized from emulsion droplets. In dispersed phase, the mixing ratio of aqueous silicic acid solution and ammonium hydroxide solution was maintained as (a) 5:1, (b) 7:1, and (c) 9:1. The precursor mixture of silicic acid and ammonium hydroxide was not diluted before emulsification. The concentration of trichlorododecylsialne dissolved in toluene was maintained as $0.12 \mathrm{ml} / \mathrm{ml}$, and dispersed phase was prepared without dilution using distilled water after mixing of silicic acid solution and ammonia solution, followed by homogenization. (d) Pore size distribution of silica aerogel particles before and after surface modification using trichlorododecylsilane synthesized using silicic acid to ammonium hydroxide solution as 9:1 mixing ratio.

implying that decreasing the $\mathrm{pH}$ value while increasing the amount of ammonia caused more rapid gelation inside the emulsion droplets. The size distribution of the spherical aerogel particles was polydisperse, since the original emulsion droplets generated by the homogenizer showed broad size distribution. Although the results are not reproduced here, the morphologies of the silica aerogel particles were also spherical when the mixing ratio was 10:1.

Fig. 3(d) contains the pore size distribution of the silica aerogel particles synthesized using the silicic acid solution and ammonium hydroxide at a mixing ratio of 9:1. Although the $\mathrm{N}_{2}$ adsorption and desorption data is not reproduced here, the BET surface area of this sample was measured to be $682.36 \mathrm{~m}^{2} / \mathrm{g}$. Although the average pore size was measured to be $3.5 \mathrm{~nm}$ by the nitrogen adsorption technique, it became

Table 1. $\mathrm{pH}$ of dispersed phase as a function of the mixing ratio of silicic acid and ammonium hydroxide.

\begin{tabular}{ccccccccccc}
\hline Mixing Ratio & $1: 2$ & $2: 1$ & $3: 1$ & $4: 1$ & $5: 1$ & $6: 1$ & $7: 1$ & $8: 1$ & $9: 1$ & $10: 1$ \\
\hline $\mathrm{pH}$ & 12 & 9 & 8 & 7 & 7 & 6.5 & 6 & 5 & 5 & 5 \\
\hline
\end{tabular}


difficult to detect pore size distribution after surface modification using trichlorododecylsilane. Since the porous surface could be decorated with dodecyl groups after modification, nitrogen adsorption may be hindered by hydrocarbon chains, which block the bare surface of the porous structure of the silica aerogel, causing a drastic change in pore size distribution before and after surface modification.

When the volume ratio of silicic acid to ammonium hydroxide solution was fixed at 9:1, the amount of trichlorododecylsilane

(a)

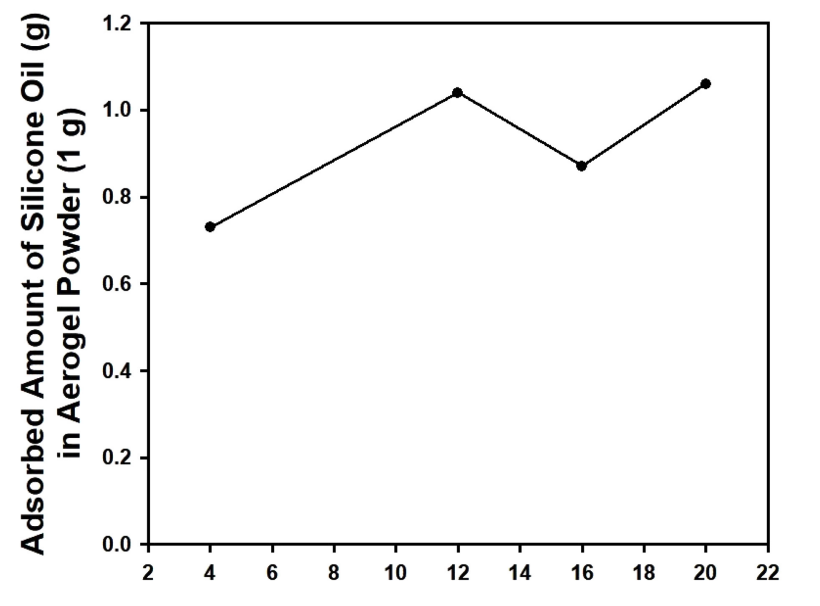

Volueme \% of trichlorododecylsilane in Toluene

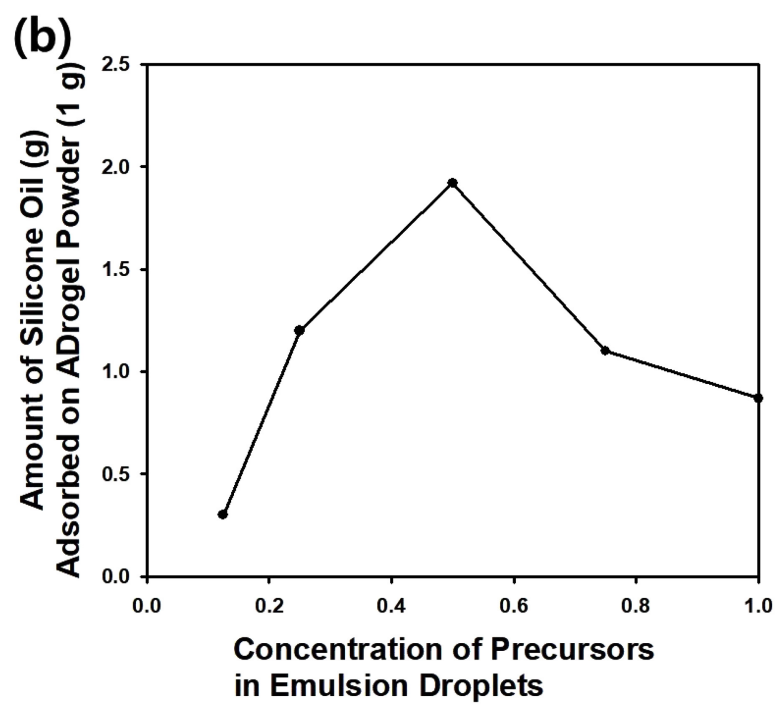

Fig. 4. Adsorbed amount of silicone oil (100 CS) as a function of (a) the amount of trichlorododecylsilane during surface modification. (b) Change of adsorbed amount of silicone oil (100 CS) as function of the concentration of precursor materials inside emulsion droplets. The mixing ratio of silicic acid and ammonia solution was maintained as 9:1 for Figure 4(a). The concentration of trichlorododecylsialne dissolved in toluene was maintained as $0.12 \mathrm{ml} / \mathrm{ml}$. was changed to investigate the effect on the amount of adsorbed silicone oil (100 CS). As displayed in Fig. 4(a), the amount of adsorbed oil increased 'in overall' with increasing amount of the silane coupling agent, since enhanced hydrophobicity can be expected with increasing number of hydrocarbon groups derived from the coupling agent. However, the amount of adsorbed silicone oil declined as the concentration of silane coupling agent dissolved in toluene was increased from 12 to $16 \%$. Since the nanopores of the aerogel particles can be blocked by chemisorption of the silane coupling agent, the amount of adsorbed oil can be reduced by excessive addition of the coupling agent. However, a further increase of the coupling agent concentration to higher than $16 \%$ may form a second hydrophobic layer on the particle surface, which may increase the amount of adsorbed oil again [22].

Unlike the effect of the silane coupling agent content on oil adsorption, there was an optimal amount of precursors in the dispersed phase (emulsion droplets) to achieve the maximum amount of adsorbed silicone oil (100 CS), as shown in Fig. 4(b). When the mixture of silicic acid and ammonium hydroxide was diluted using the same volume of distilled water, the amount of adsorbed silicone oil was measured at its maximum value, indicating that too diluted or concentrated precursors resulted in a decrease in the amount of adsorbed oil. Accordingly, it is thought that an optimal value exists in the void spaces to contain liquid oil in the solid silica phase modified with hydrophobic silane coupling agent in aerogel particles.

In this study, trichloromethylsilane, with its shorter hydrocarbon group, was also used to modify the aerogel particles to obtain a spherical hydrophobic powder, as shown in Fig. 5(a). The amount of silicic acid mixed with ammonium hydroxide solution was adjusted to determine the composition of precursor solution for optimal oil adsorption capacity after the modification using trichloromethylsilane, As shown in Fig. 5(b), the surface area decreased from 733.46 to $246.19 \mathrm{~m}^{2} / \mathrm{g}$ after modification using the silane coupling agent, implying that chemisorption of the trichloromethylsilane caused blocking of the nanopores in the aerogel particles. As shown in the inset graph of Fig. 5(b), the average pore size before surface modification was measured to be $88.23 \AA$, and the very sharp distribution of the pore size disappeared after modification. However, a new broad pore 


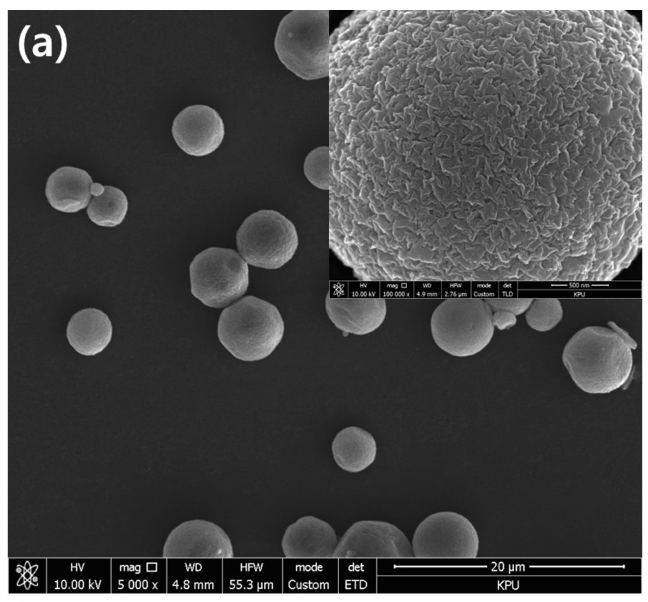

(b)

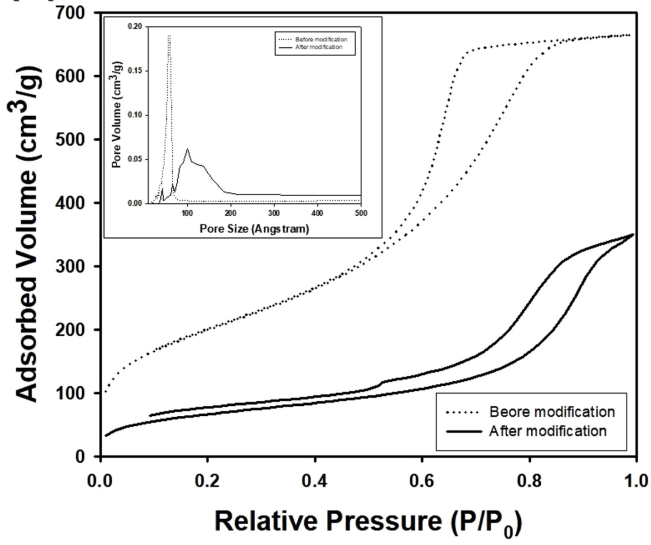

(c)

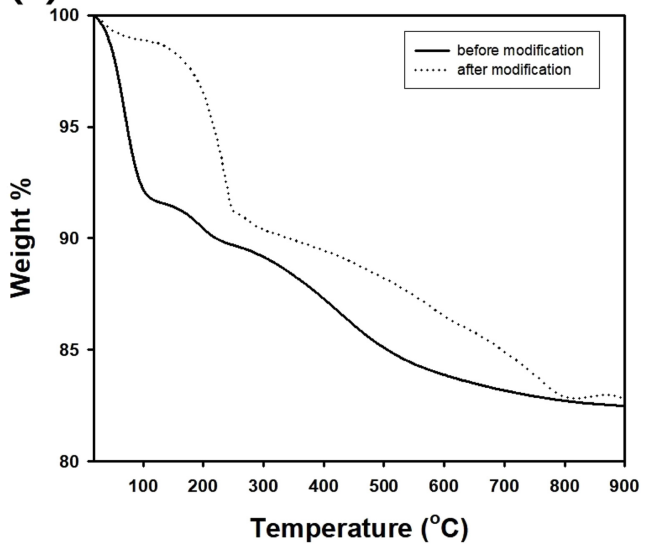

Fig. 5. (a) SEM image of silica aerogel particles fabricated using precursor mixture of 8:1 mixing ratio of silicic acid and ammonium hydroxide solution. The precursor mixture of silicic acid and ammonium hydroxide was not diluted before emulsification. (b) BET data of silica aerogel particles fabricated using precursor mixture of 8:1 mixing ratio of silicic acid and ammonium hydroxide solution. BET analyses were carried out before and after modification using trichloromethylsilane. Inset figure shows pore size distribution of the aerogel particles before and after surface modification. (c) TGA results of silica aerogel particles fabricated using precursor mixture of 8:1 mixing ratio of silicic acid and ammonium hydroxide solution. before and after surface modification using trichloromethylsilane. size distribution from 10 to $20 \mathrm{~nm}$ appeared after the surface modification, similar to porous titania nanoparticles modified with 3-(2-aminoethylamino)propyldimethoxymethylsilane [23]. Although the origin of the broad peak formation is not very clear, it is thought that nanopores can be filled by the silane coupling agent, leading to the formation of rough surfaces by successive chemisorption of the coupling agent, causing pore-like structures on the particle surface.

Fig. 5(c) contains the TGA results of the silica aerogel particles before and after surface modification using trichloromethylsilane. Because the surface methyl groups originating from the silane coupling agent can be removed by thermal degradation at temperatures higher than about $250{ }^{\circ} \mathrm{C}$, the mass of the aerogel powder modified with the silane coupling agent was heavier than that of the bare aerogel particles, as shown in Fig. 5(c). Materials remaining after the decomposition of hydrocarbon were recorded up to a high temperature of $800^{\circ} \mathrm{C}$, implying that a carbon component may be coated on the aerogel particles after heat treatment at high temperature.

To determine the optimum synthesis conditions for oil adsorption, the mixing ratio of silicic acid to ammonium hydroxide solution was adjusted from $5: 1$ to $10: 1$ before the emulsification step. As shown in Fig. 6(a), an optimum value of the mixing ratio was found for maximum oil adsorption. Among several samples, the aerogel powder with the 8:1 mixing ratio in the SEM image of Fig. 5(a) showed the maximum adsorption capacity, as displayed in the graph of Fig. 6(a). Like the aerogel powder modified with trichlorododecylsilane, the optimal mixing ratio of precursors was determined to be $8: 1$, implying that mixing ratios from 7:1 to 8:1 can result in the largest amount of oil adsorption regardless of the molecular structure of the silane coupling agent. This indicates that oil adsorption can be maximized using particles with a mixture of disk-shaped and spherical morphologies. When irregular-shaped or spherical particles were solely used, oil adsorption was less effective than the adsorption capacity of aerogel particles.

The random packing of the mixture of spherical aerogel particles and disk-shaped aerogel particles can be modeled as a mixture of spheres and rod-like particles with a small aspect ratio (L/D). Since the random packing fraction of this mixture is higher than that of pure spheres $(0.634)$, more compact packing of the mixed particles can be expected, 
causing enhanced oil adsorption per unit volume of powder [24]. Thus, it is advantageous to use aerogel particles prepared at mixing ratios from 7:1 to $8: 1$ (aqueous silicic acid : ammonia solution).

The amount of trichloromethylsilane was also changed to investigate the effect on oil adsorption capacity after surface modification of the aerogel powder, as displayed in Fig. 6(b). When the amount of hydrophobizing agent, trichloromethylsilane was too small, the amount of adsorbed silicone oil was smaller than $200 \%$ due to insufficient coverage of the methyl groups on the surface of the aerogel particles, which means the resulting silica aerogel particles adsorbed silicone oil (100 CS) equal to almost 2 times the mass of the porous particles. Because the nanopores contained in the aerogel particles can be blocked to prevent liquid oil from being absorbed into the particles, an excessive amount of trichloromethylsilane also resulted in a decrease in the amount of silicone oil adsorbed. Thus, there was an optimal amount of trichloromethylsilane needed to achieve the maximum amount of oil adsorption, $250 \%$, as displayed in Fig. 6(b).

The specific surface area of the silica aerogel particles fabricated and modified under optimal conditions was measured by BET method, and the nitrogen adsorption and desorption results are shown for samples before and after modification using trichloromethylsilane.

In this study, silicone oils with various viscosities were used to measure the amount of oil adsorbed by silica aerogel particles synthesized by silicic acid solution and ammonium hydroxide at an 8:1 mixing ratio and subsequent modification using trichloromethylsilane. Overall, the amount of oil adsorbed decreased as the viscosity of the silicone oil increased, as shown in Fig. 6(c). Since viscous oil is difficult to infiltrate through the porous structure of the aerogel, a highly viscous liquid resulted in less adsorption by aerogel particles. This can be explained by the following Washburn equation [25].

$$
h^{2}=0.25 r_{e f f}^{2} \Delta P t / \eta
$$

Here, $h$ and $r_{\text {eff }}$ indicate the infiltration height through pores and the effective radius of the pore, respectively. $\Delta P$ stands for the pressure difference between the applied and critical pressure, whereas $t$ and $\eta$ are the infiltration time and the (a)

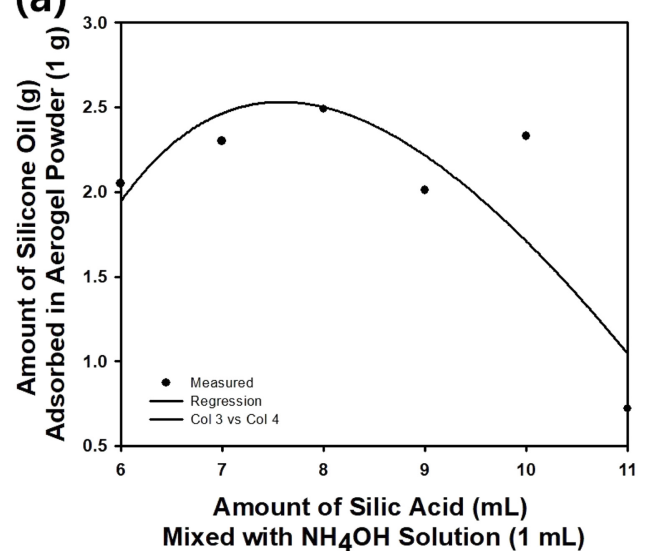

(b)

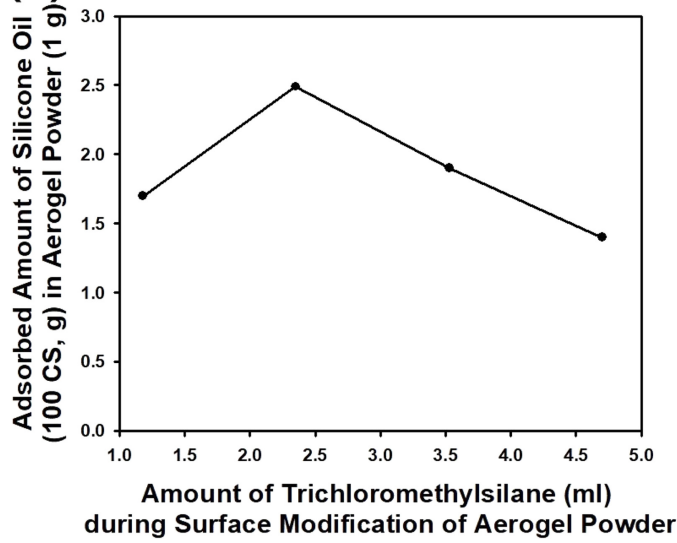

(c)

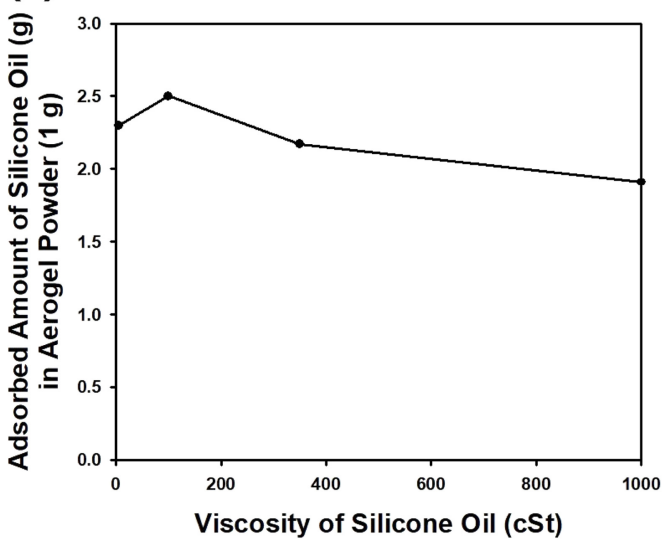

Fig. 6. (a) Adsorbed amount of silicone oil (100 CS) as a function of the amount of silicic acid solution mixed with ammonium hydroxide solution $(1 \mathrm{ml})$ in dispersed phase. Silica aerogel particles were modified using trichloromethylsilane $(0.047 \mathrm{ml} / \mathrm{ml})$. (b) Adsorbed amount of silicone oil (100 CS) as a function of the amount of trichloromethylsilane. Silica aerogel particles were fabricated using precursor mixture of $8: 1$ mixing ratio of silicic acid and ammonium hydroxide. The precursor mixture of silicic acid and ammonium hydroxide was not diluted before emulsification. (c) Change of adsorbed amount of silicone oil by silica aerogel particles modified with trichloromethylsilane as a function of viscosity of the oil. 
(a)
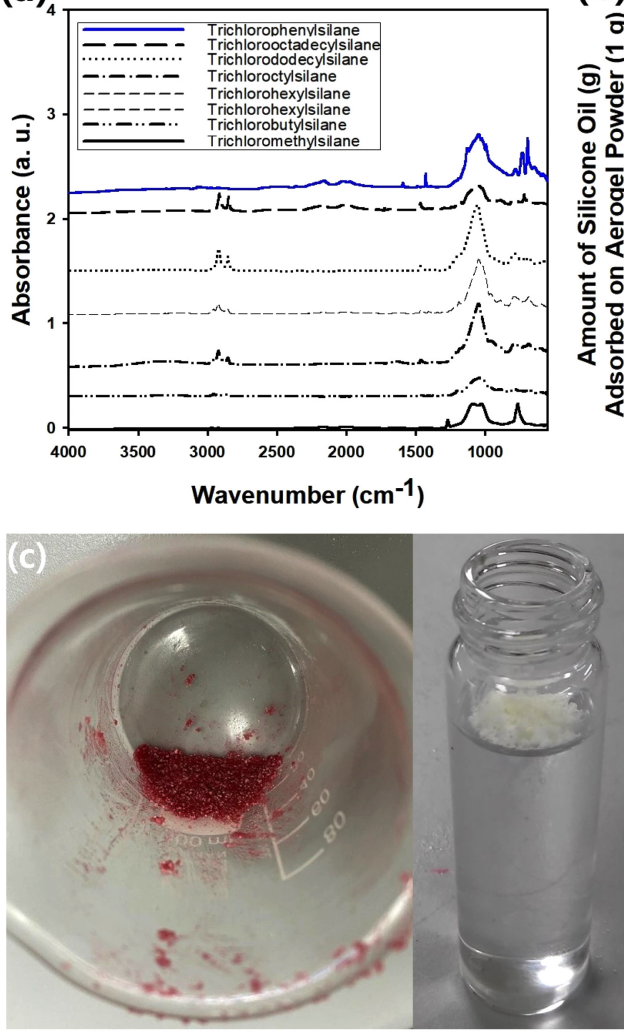

(b)

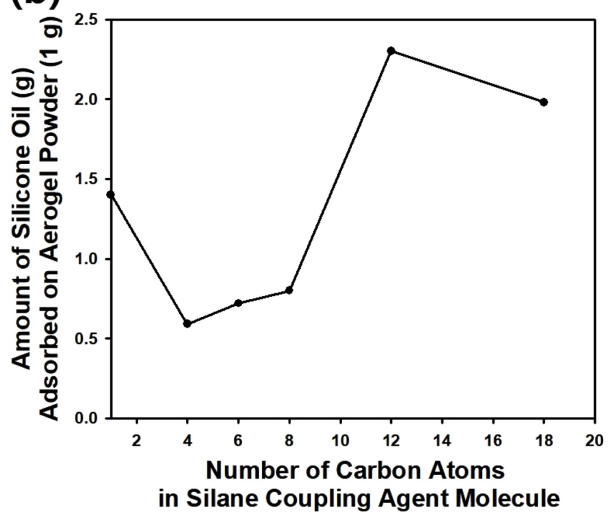

(d)

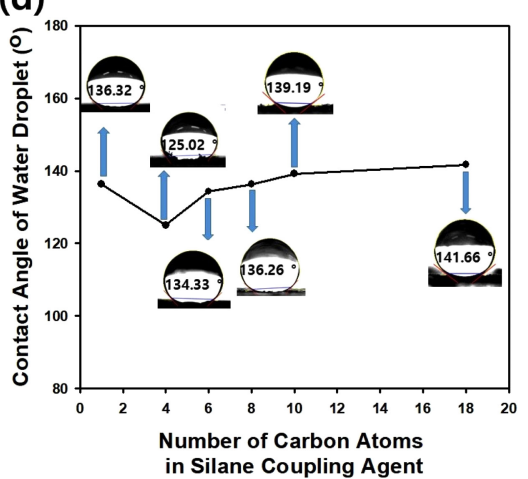

Fig. 7. (a) FT-IR spectrum of silica aerogel particles after surface modification using various silane coupling agents such as trichlorophenylsilane, trichlorooctadecylsilane, trichlorododecylsilane, trichlorohexylane, and trichloromethylsilane. (b) Adsorbed amount of silicone oil (100 CS) using aerogel powder fabricated under optimal conditions after surface modification using different kinds of silane coupling agents. (c) Photograph of silica aerogel after oil adsorption (left) and photograph of silica aerogel particles on water-air interface (right). (d) Contact angle of water droplet on coating film of silica aerogel particles modified with various kinds of silane coupling agents.

viscosity of the liquid, respectively. Thus, infiltration height decreases with increasing liquid viscosity, causing a decrease in amount of adsorbed silicone oil, since the infiltration of a viscous liquid through the nanopores of the aerogel particle is difficult due to the existence of surface anchoring groups originating from the silane coupling agent.

In this study, various kinds of silane coupling agents with alkyl groups having different length of hydrocarbon chains were adopted for surface modification of the silica aerogel particles. Fig. 7(a) contains the FT-IR spectra of the silica aerogel particles after modification with trichloromethylsilane, trichlorohexylsilane, trichlorododecylsilane, and trichlorooctadecylsilane. When the modification was performed using trichloromethylsilane, the FT-IR spectrum of the resulting aerogel particles was similar to PDMS (polydimethylsiloxane), indicating that the characteristic peaks appeared at about 790 and $1,030 \mathrm{~cm}^{-1}$ due to the Si-C stretching of S-CH . The peak that appeared at about $1,260 \mathrm{~cm}^{-1}$ was derived from the $\mathrm{CH}_{3}$ deformation of $\mathrm{Si}_{-} \mathrm{CH}_{3}$. [26]. Although similar characteristic peaks appeared in the FT-IR spectra of the silica aerogel particles treated with trichlorohexylsilane and trichlorododecylsilane, the peak intensity from trichlorododecylsilane was stronger compared to that of trichlorohexylsilane, indicating that longchain hydrocarbon resulted in a more intensive absorption peak. Characteristic peaks at similar wavenumber appeared from the silica aerogel powder modified with trichlorooctadecylsilane, as shown in Fig. 7(a). The FT-IR spectrum of the silica aerogel powder modified with trichloropheylsilane was also measured, and the result is shown as a blue line in Fig. 7(a), indicating the characteristic peaks appeared at 1427, 696, and $727 \mathrm{~cm}^{-1}$ due to the $\mathrm{Si}_{-} \mathrm{C}_{6} \mathrm{H}_{5}$ bond [27].

Fig. 7(b) contains the change in the amount of silicone oil (100 CS) adsorbed by the silica aerogel particles as a function of the number of carbon atoms in the silane coupling 
agent used for surface modification of the particles. From the graph in Fig. 7(b), it is evident that the adsorbed amount of oil increased with increasing number of carbon atoms in the silane coupling agent, except for the result from trichloromethylsilane, since larger alkyl groups can enhance the hydrophobicity of the aerogel particles.

However, silica aerogel particles modified with trichlorooctadecylsilane revealed less oil adsorption compared to the sample modified with trichlorododecylsilane, because bulky hydrocarbon chains may block the nanopores in the aerogel particles, causing a decreased in oil adsorption. When trichloromethylsilane was used for surface modification, the amount of silicone oil adsorbed was larger than that of trichlorobutylsilane, since the blocking of nanopores can be avoided by using trichloromethylsilane with an alkyl chain shorter than the other types of silane coupling agent. Though the result is not included in Fig. 7(b), silica aerogel particles modified with trichlorophenylsilane showed an adsorption capacity of $131.7 \%$, which is a larger value than that of the aerogel powder treated with trichlorohexylsilane, $72 \%$. It is thought that this large discrepancy was caused by the difference in the molecular structures of trichlorophenylsilane and trichlorohexylsilane, implying that aromatic groups can be more advantageous than aliphatic groups for the adsorption of oil.

In the photograph in Fig. 7(c), silica aerogel particles adsorbing the silicone oil can be seen to be a solid-like phase, indicating that the saturated state was achieved without an unadsorbed liquid-phase oil. For better visualization, the oil was stained red using Sudan III dye. Since the aerogel particles are lighter than water because of their porous and hydrophobic nature after surface modification, the particles floated on the water-air interface, as shown in the photograph in Fig. 7(c). The degree of hydrophobicity of the aerogel particles was assessed by measuring the contact angle of a water droplet coating a film composed of the particles, as displayed in the photograph in Fig. 7(d). As the number of carbon atoms in the hydrocarbon chain of the silane coupling agent used during surface modification increased from 4 to 18 , the contact angle increased from 125.02 to $141.66^{\circ}$, indicating that more hydrophobic particles could be prepared using the silane coupling agent with longer alkyl chains. However, the contact angle of the trichloroalkyl silane was
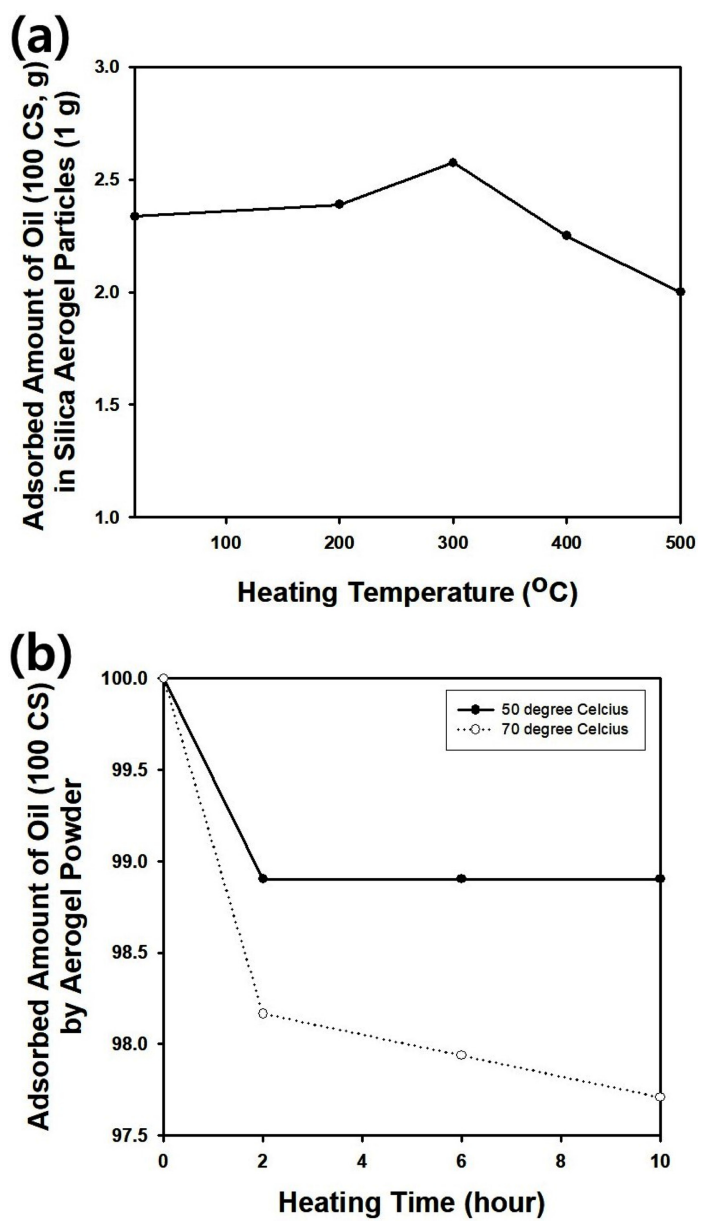

Fig. 8. Adsorbed amount of silicone oil (100 CS) per $1 \mathrm{~g}$ of aerogel particle (a) as a function of heating temperature after surface modification using trichloromethylsilane and (b) heating time at 50 and $70{ }^{\circ} \mathrm{C}$ after oil adsorption.

higher than those of the coupling agent with butyl, hexyl, and octyl groups, because the larger pore volume of the aerogel particles may remain after modification with the short methyl groups, unlike the other types of silane coupling agents with longer alkyl chains.

Fig. 8(a) shows the change in oil adsorption capacity of the silica aerogel particles modified with trichloromethylsilane as a function of the heating temperature of the particles before oil adsorption. When the heating temperature was lower than $300{ }^{\circ} \mathrm{C}$, the adsorption of silicone oil was enhanced, growing with increasing heat treatment temperature. This indicates that carbon elements derived from the coupling agents may remain on the surface of the aerogel particles after the heat treatment, to maintain the hydrophobicity of the particles. 
Though oil adsorption should be enhanced with increasing temperature, it decreased again at higher temperature, since the short hydrocarbon chains derived from the trichloromethylsilane were easily decomposed by applying larger thermal energy. In this way, the optimal heating temperature was determined to be about $300{ }^{\circ} \mathrm{C}$ for aerogel particles modified with trichloromethylsilane.

Fig. 8(b) illustrates the change in the oil adsorption capacity as a function of heating time after oil adsorption. The measurement was carried out using silica aerogel particles synthesized under optimal conditions and modified using trichloromethylsilane. Due to the diffusion of silicone oil adsorbed by the aerogel particles, oil adsorption capacity decreased with increasing heating time at a relatively high temperature. However, the amount of oil adsorption decreased and became negligible after 2 hours of heating at $50{ }^{\circ} \mathrm{C}$, indicating that a prolonged heating time at a higher temperature like $70{ }^{\circ} \mathrm{C}$ is necessary to remove adsorbed oil from the particles.

In this study, silica aerogel particles synthesized from emulsion droplets were applied as an oil adsorber to prepare composite films containing lubricant oil to avoid ice adhesion to the film surface. In winter season, ice can be formed on construction surfaces under humid air. Because this may reduce the generation rate of electricity by depressing the motion of blades in a wind power system, an adequate coating technique is essential to detach the adhered ice layer. Since our hydrophobic aerogel particles containing a large amount of oil can be mixed with polymer films like PDMS, they can be applied as an icephobic coating.

Icephobicity can be quantitatively discussed using ice adhesion strength, $\tau_{\text {ice}}$, which can be defined as the shear stress needed to detach an adhered ice layer from a surface. Fig. 9(a) shows the change in the ice adhesion strength $\left(\tau_{\text {ice }}\right)$ of composite films made of PDMS, silica aerogel particles, and silicone oil as a function of the amount of particle-oil mixture. The viscosity of the oil was $100 \mathrm{cSt}$, and the mixing ratio of the oil and the aerogel particles modified with trichloromethyl silane was maintained at 1:0.3. Because the oil functions as a lubricant it can be enhanced by increasing the amount of particle-oil mixture, and $\tau_{\text {ice }}$ could be reduced with increasing amount of the particle-oil mixture in the film, implying that adhered ice layer cannot be maintained on the
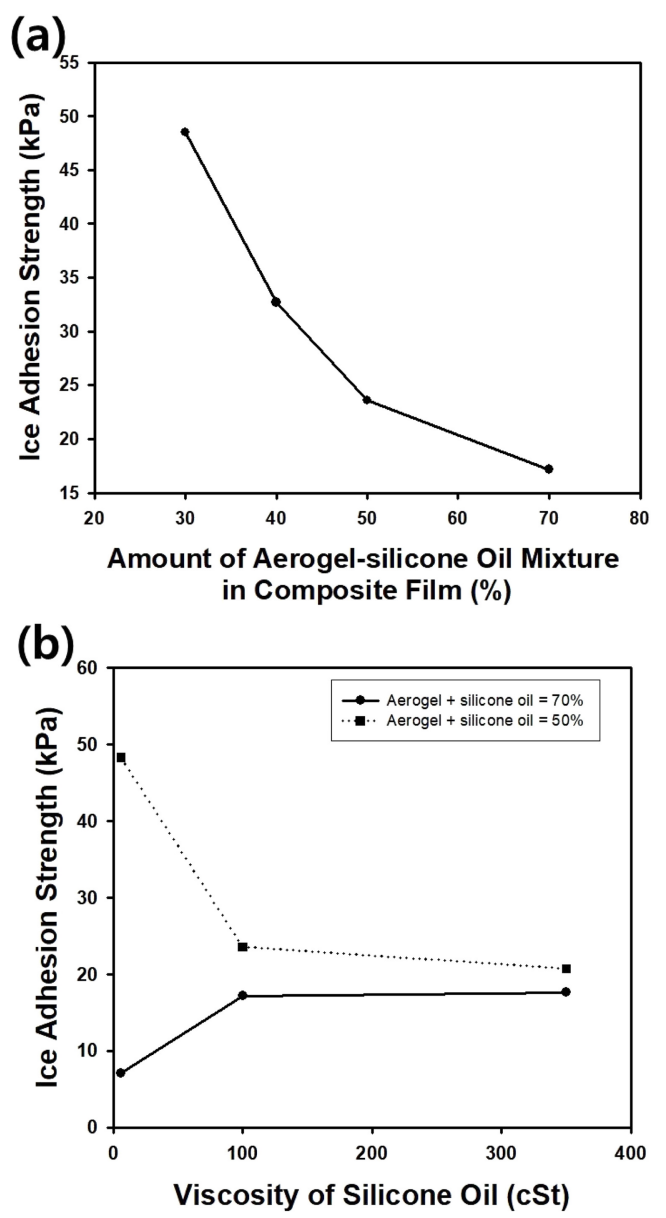

Fig. 9. Ice adhesion strength of composite film as function of (a) the amount of aerogel-silicone oil mixture and (b) the viscosity of silicone oil. Silica aerogel particles modified with trichloromethylsilane was adopted to prepare particle-oil mixture.

surface for long time.

Fig. 9(b) shows the change in ice adhesion strength as a function of the viscosity of the silicone oil mixed with silica aerogel particles. When the weight percent of the particle-oil mixture was $70 \%, \tau_{\text {ice }}$ increased as the viscosity of oil increased, indicating that solid particles embedded in the composite film may increase the surface roughness of the film, causing strong attachment of the ice layer to the film surface. However, $\tau_{\text {ice }}$ decreased for the composite film with a $50 \%$ particle-oil mixture with increasing oil viscosity, due to the slow elution of viscous oil from the particles.

Fig. 10(a), 10(b), and 10(c) contain SEM images of the silica microparticles with wrinkled surfaces, synthesized using silicic acid solution and distilled water at different mixing ratios of $6: 1,4: 3$, and 1:6, respectively. As the amount 

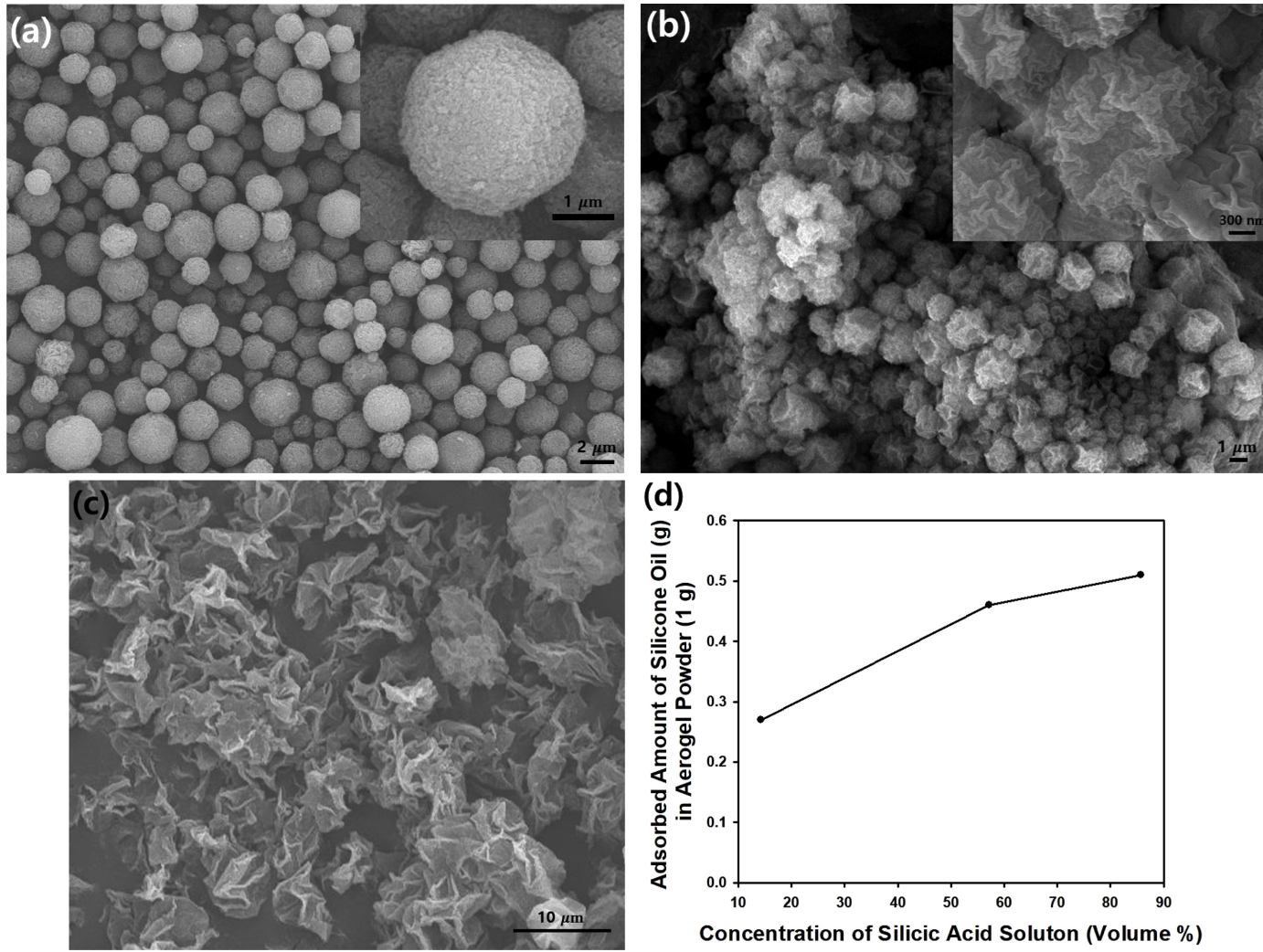

Fig. 10. SEM image of silica particles with wrinkled surfaces. The mixing ratio of silicic acid and water was changed as (a) $6: 1$, (b) $4: 3$, and (c) 1:6. (d) Adsorbed amount of silicone oil $(100 \mathrm{CS})$ using silica particles with wrinkled surfaces. The concentration of trichlorododecylsialne dissolved in toluene was maintained as $0.12 \mathrm{ml} / \mathrm{ml}$.

of water in the dispersed phase (emulsion droplets) increased, the volume occupied by the silica material in the final particles decreased, due to decrease in silica precursor during the synthesis step, whereas a high concentration of silicic acid resulted in more compact particles, as displayed in the SEM images in Fig. 10(a) to 10(c).

As shown in the graph in Fig. 10(d), the adsorbed amount of silicone oil (100 CS) increased with the increasing concentration of silicic acid, indicating that a greater amount of oil molecules could be adhered more strongly to the compact silica particles with wrinkled surfaces shown in Fig. 10(a), compared to the crumpled silica microparticles with less dense structure in Fig. 10(b) or 10(c). Unlike the aerogel particles with a nanoporous structure, the porous nature of the silica microparticles shown in Fig. 10(a) to 10(c) can be considered 'macroporous' particles with irregular-shaped pores. Since macropores are much larger than nanopores, huge amounts of liquid oil may not be contained in the macropores due to the difference in the specific surface areas of the aerogel and macroporous particles. To confirm this, the BET surface area of the silica microparticles in Fig. 10(b) was measured to be $148.93 \mathrm{~m}^{2} / \mathrm{g}$, which is much smaller than that of the silica aerogel particles shown in Fig. 3 or Fig. 7, indicating that the specific surface area strongly affects the amount of adsorbed oil.

Porous structures can be expected to have enhanced properties including mechanical properties [29]. In this study, the morphologies as well as porous structure of the silica

Table 2. Synthesis conditions of PS nanospheres used as templating materials for porous silica microparticles.

\begin{tabular}{ccccccc}
\hline Sample & Styrene & Water & KPS & NaSS & Polymerization Temperature & Particle Size (nm) \\
\hline$\# 1$ & $43 \mathrm{ml}$ & $380 \mathrm{ml}$ & $0.196 \mathrm{~g}$ & $0.49375 \mathrm{~g}$ & $70{ }^{\circ} \mathrm{C}$ & 230 \\
$\# 2$ & $43 \mathrm{ml}$ & $380 \mathrm{ml}$ & $0.196 \mathrm{~g}$ & $0.1234 \mathrm{~g}$ & $70{ }^{\circ} \mathrm{C}$ & 560 \\
\hline
\end{tabular}



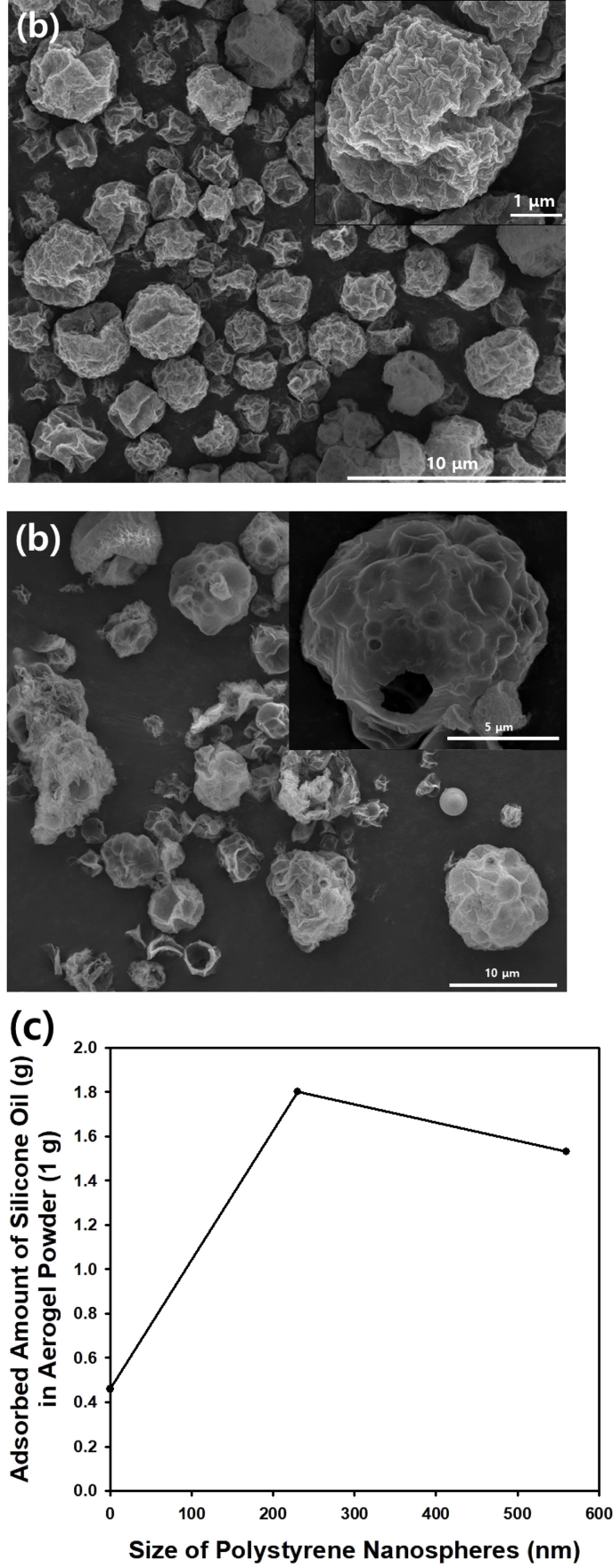

Fig. 11. SEM image of porous silica particles with wrinkled surfaces. The size of templating material, PS nanospheres was (a) 230 and (b) $560 \mathrm{~nm}$. (d) Adsorbed amount of silicone oil (100 CS) using porous silica particles with wrinkled surfaces shown in SEM image of Figure 11(a) and (b). The concentration of trichlorododecylsialne dissolved in toluene was maintained as $0.12 \mathrm{ml} / \mathrm{ml}$.

microparticles with wrinkled surfaces could be changed by the addition of PS nanospheres as templating materials in a dispersed phase during synthesis. After calcination, porous silica microparticles with hollow interior structures were fabricated after removal of the PS nanospheres, due to removal of the polymeric beads, as shown in the SEM images of Fig. 11(a) and 11(b). For comparison, the amount of silicone oil adsorbed (100 CS) was measured using the porous silica microparticles shown in Fig. 11(a) and 11(b), as contained in the graph of Fig. 11(d). Since the porous structures derived from the PS templates provide more space for oil adsorption, the amount of adsorbed oil increased when the PS nanospheres were included inside the emulsion droplets. However, the oil adsorption capacity of the porous silica particles was inferior to that of silica aerogel particles, because the aerogel particles contained smaller nanopores [28]. The BET surface area of porous silica particles in Fig. 11(b) was measured to be $608.3 \mathrm{~m}^{2} / \mathrm{g}$, which is smaller than that of the silica aerogel particles, and therefore the adsorption capacity was inferior to the results of the aerogel particles.

\section{Conclusions}

In this study, silica aerogel particles were synthesized inside emulsion droplets as micro-reactors using sodium silicate as an economic raw material. After gelation at room temperature under ambient pressure, solvent exchange steps were carried out to maintain the porous structures of the particles, followed by surface modification using silane coupling agent to prepare hydrophobic particles. Factors affecting the adsorbed amount of silicone oil were studied to determine the optimal synthesis conditions of the aerogel particles. Morphologies of the aerogel were changed from irregular-shaped, disk-type, and spherical by increasing the concentration of ammonium hydroxide, and an optimal mixing ratio of silicic acid to ammonia solution (8:1) to maximize oil adsorption capacity was determined. The concentration of precursor materials in the dispersed phase also affected the adsorption capacity and the adequate dilution factor of the reactants was determined, indicating that too concentrated or diluted precursors resulted in less adsorption capacity.

Rough surfaces with irregular protrusions of several tens of nanometer were observed on the aerogel particles synthesized by optimal conditions. The maximum adsorption capacity of 
silicone oil $(100 \mathrm{CS})$ was measured to be $250 \%$ with respect to the weight of the aerogel particles, when trichloromethylsilane was used as a surface modifying agent. However, the adsorbed amount of oil varied for aerogel particles modified with other types of coupling agent with different hydrocarbon chain lengths, due to changes in the hydrophobicity of the particles.

\section{Acknowledgments}

This research was supported by Priority Research Centers Program through the National Research Foundation of Korea (NRF) funded by the Ministry of Education (NRF2017R1A6A1A03015562), the Industrial core technology development program (10077545, Development of icephobic coating materials for extreme environment) funded by the Ministry of Trade, industry \& Energy(MI, Korea), and Korea Institute for Advancement of Technology(KIAT) grant funded by the Korea Government(MOTIE) (P0002007, The Competency Development Program for Industry Specialist). Y.-S. Cho thanks to the support from Korea Polytechnic University during his research year in 2020 .

\section{REFERENCES}

1. S. Ould-Chikh, S. Pavan, A. Fecant, E. Trela, C. Verdon, A. Gallard, N. Crozet, J.-L. Loubet, M. Hemati, and L. Rouleau, Stud. Surf. Sci. Catal. 175, 193 (2010).

2. P. Ruckdeschel, A. Philipp, and M. Retsch, Adv. Funct. Mater. 27, 1702256 (2017).

3. M. Beaumont, B. Buchegger, S. F. Plappert, J.-M. Nedelec, M. Opietnik, A. Potthast, and T. Rosenau, Cellulose 26, 683 (2019).

4. Klaus Unger, Angew. Chem. Int. Edit. 11, 267 (1972).

5. D. Zhang, A. Forner-Cuenca, O. O. Taiwo, V. Yufit, F. R. Brushett, N. P. Brandon, S. Gu, and Q. Cai, J. Power Sources 447, 227249 (2020)

6. R. L. Thankamony, X. Li, X. Fan, G. Sheng, X. Wang, S. Sun, X. Zhang, and Z. Lai, ACS Appl. Mater. Interf. 10, 44041 (2018).

7. E. Liu, H. Zhao, H. Li, G. Li, Y. Liu, and R. Chen, New J. Chem. 38, 2911 (2014).

8. C. J. Brinker, R. Sehgal, S. L. Hietal, R. Deshpande, D. M. Smith, D. Loy, and C. S. Ashley, J. Membrane Sci. 94, 85
(1994).

9. P. H. Kim and K. Y. Jung, RSC Adv. 6, 1686 (2016).

10. V. Singh, C. Cassidy, F. Abild-Pedersen, J.-H. Kim, K. Aranishi, S. Kumar, C. Lal, C. Gspan, W. Grogger, and M. Sowwan, Nanoscale 7, 13387 (2015).

11. M. A. Hasan, R. Sangashetty, A. C. M. Esther, S. B. Patil, B. N. Sherikar, and A. Dey, J. Inst. Engineer D 98, 297 (2017).

12. J. Choi and D. J. Suh, Catal. Surv. Asia 11, 123 (2007).

13. L.W Hrubesh and J. F Poco, J. Non-Cryst. Solids 188, 46 (1995).

14. J. Quiño, M. Ruehl, T. Klima, F. Ruiz, S. Will, and A. Braeuer, J. Supercrit. Fluid. 108 (2016).

15. Y.-S. Cho, I.-A. Oh, and N. R. Jung, J. Disper. Sci. Technol. 37, 676 (2016).

16. H. J. Kim, S. W. Han, J. H. Kim, H. O. Seo, and Y. D. Ki, Curr. Appl. Phys. 18, 369 (2018).

17. K. Okiel, M. El-Sayed, and M. Y. El-Kady, Egypt. J. Petrol. 20, 9 (2011).

18. X. Li, Y. Du, Z. Li, G. Wu, H. Li, and H. Sui, Chemosphere, 88, 245 (2012).

19. C. A. Franco, M. Martínez, P. Benjumea, E. Patiño, and F. B. Cortés, Adsorp. Sci. Technol. 32, 197 (2014).

20. Y.-S. Cho, N. H. Ku, and Y. S. Kim, J. Chem. Eng. Jpn. 52, 194 (2019).

21. Y.-S. Cho, S. Jeong, and S. Nam, Korean J. Met. Mater. 58 $1(2020)$.

22. C. H. Zanchi, F. A. Ogliari, R. M. e Silva, R. G. Lund, H. H. Machado, C. Prati, N. L. V. Carreño, and E. Piva, Appl. Adhesion Sci. 3, 27 (2015).

23. A. R. M. Dalod, L. Henriksen, T. Grande, and M.-A. Einarsrud, Beilstein J. Nanotechnol. 8, 304 (2017).

24. A. V. Kyrylyuk, A. Wouterse, and A. P. Philipse, AIP Conference Proceedings 1145, 211 (2009).

25. N. Eustathopoulos, Metals 5, 350 (2015).

26. L. M Johnson, L. Gao, C. W. Shields IV, M. Smith, K. Efimenko, K. Cushing, J. Genzer, and G. P López, J. Nanobiotechnol. 11, 22 (2013).

27. E. N. Ermakova, S. V. Sysoev, L. D. Nikulina, I. P. Tsyrendorzhieva, V. I. Rakhlin, and M. L. Kosinova, Mod. Electron. Mater. 1, 114 (2015).

28. Y.-S. Cho and J. W. Moon, Arch. Metall. Mater. 62, 1371 (2017).

29. F. Chen, H. Wang, Q. Shen, E. J. Lavernia, and L. Zhang, Met. Mater. Int. 25, 83 (2019). 